\title{
Baryons in the Field Correlator Method: Effects of the Running Strong Coupling
}

\author{
R. Ya. Kezerashvili ${ }^{1}$, I. M. Narodetskii ${ }^{1,2}$, A. I. Veselov ${ }^{2}$ \\ ${ }^{1}$ Physics Department, The City University of New York, New York, 11201, USA \\ ${ }^{2}$ Institute of Theoretical and Experimental Physics, Moscow 117218, Russia
}

\begin{abstract}
The ground and $P$-wave excited states of $n n n, n n s$ and $s s n$ baryons are studied in the framework of the field correlator method using the running strong coupling constant in the Coulomb-like part of the three-quark potential. The running coupling is calculated up to two loops in the background perturbation theory. The three-quark problem has been solved using the hyperspherical functions method. The masses of the $S$ - and $P$-wave baryons are presented. Our approach reproduces and improves the previous results for the baryon masses obtained for the freezing value of the coupling constant. The string correction for the confinement potential of the orbitally excited baryons, which is the leading contribution of the proper inertia of the rotating strings, is estimated.
\end{abstract}

\section{Introduction}

Quantum chromodynamics (QCD) has been established as the theory describing the strong interaction but its application to low-energy hadron phenomenology is still far from a routine deduction. Various approximations, whose connection to the underlying theory remains sometimes obscure, are presently used to describe baryon spectroscopy. There is a vast literature of theoretical treatment of baryons, including treatment in specific quark models [1], QCD sum rules [2], effective field theories [3], the Skyrme model 44, the collective models of the baryon such as the algebraic approach of Ref. [5], and large $N_{c}$ analysis [6], as well as on the lattice [7. It has become an attractive program to develop model independent methods which are firmly based in fundamental theory. In particular, ground state spectroscopy in lattice QCD calculations appears to be well understood [8]. Excited state spectroscopy on the lattice, however, is still a challenging task 9 .

The field correlator method (FCM) in QCD [10] provides another perspective. The FCM is a promising formulation of the nonperturbative QCD that gives additional support to the quark model assumptions. Progress was recently made [1]-14 towards placing the computation of baryon masses within the FCM on the same level as that of mesons [15. Nevertheless, this work can be refined. In Refs. [11]-14] a freezing value of the strong coupling constant in the perturbative Coulomb-like potential has been employed. This choice appears to be a reasonable approximation and gives rise to a good description of heavy quarkonia [16] and heavy-light mesons [17]. Note that within QCD most of the baryon mass is produced by quark confinement. For light baryons the Coulomb-like force does not play a crucial role and produces only a marginal $(\sim 10 \%)$ correction [1]. Nevertheless, it is important to include into the FCM approach the modern knowledge about the one-gluon exchange forces that still represent a fundamental concept which might give us a deeper understanding of baryon spectroscopy [18.

The objective of this paper is to explore the FCM for baryons in a more regular way by considering the effects of the running strong coupling constant in the Coulomb-like part of the three-quark potential. We use the background perturbation theory (BPTh) 19] for the coupling constant to avoid the infrared singularities. Below are considered the baryons composed of valence light quarks, the up, down, and strange flavors. We present the new results for the masses of the ground states and $P$-wave excited states of $n n n$, $n n s$ and ssn baryons. We also for the first time estimate the string correction to the baryonic orbital excitations. So far, this correction was calculated for the orbitally excited mesons [20, 17] and hybrid charmonium states 21 .

This paper is organized as follows. In Sec. 2, we give a brief summary of the effective Hamiltonian (EH) method important for our particular calculation 1]. In Sec. 3, we discuss the calculation of the string

\footnotetext{
${ }^{1}$ The application of this method for the baryons was described in detail elsewhere [11, 12, 14].
} 
correction. In Sect. 4, we use BPTh for the strong coupling constant to define the Coulomb-like potentials. A brief description of the hyperspherical approach, which is a very effective numerical tool for solving the three-quark problem is given in Sect. 5. Our results for the masses of the nnn, nns and ssn baryons including comparison to the previous ones obtained within the FCM with a freezing value of the strong coupling constant are presented in Sec. 6. The concluding remarks follow in Sec. 7.

\section{Effective Hamiltonian in FCM}

In the FCM three-quark dynamics in a baryon is encoded in gluonic field correlators which are responsible for quark confinement. Starting from the Feynman-Schwinger representation for the quark and gluon propagators in the external field, one can extract hadronic Green's functions and calculate the baryon spectra. The QCD string model corresponds to the limit of a small gluonic correlation length of field correlators.

The key ingredient of the FCM is the use of the auxiliary fields (AF) initially introduced in order to get rid of the square roots appearing in the relativistic Hamiltonian. Historically the AF formalism was first introduced in Ref. 22 to treat the kinematics of the relativistic spinless particles. Using the AF formalism allows one to derive a simple local form of the $\mathrm{EH}$ for the three-quark system 2 , which comprises both confinement and relativistic effects, and contains only universal parameters: the string tension $\sigma$, the strong coupling constant $\alpha_{s}$, and the bare (current) quark masses $m_{i}$. In this paper, we do not consider the spin-dependent part of the $\mathrm{EH}^{3}$. Then the $\mathrm{EH}$ has the form

$$
H=\sum_{i=1}^{3}\left(\frac{m_{i}^{2}}{2 \mu_{i}}+\frac{\mu_{i}}{2}\right)+H_{0}+V .
$$

In Eq. (11) $H_{0}$ is the nonrelativistic kinetic energy operator for the constant AF $\mu_{i}$, the spin-independent potential $V$ is the sum of the string potential

$$
V_{Y}\left(\mathbf{r}_{1}, \mathbf{r}_{2}, \mathbf{r}_{3}\right)=\sigma r_{\min },
$$

with $r_{\min }$ being the minimal string length corresponding to the Y-shaped configuration, and a Coulomb interaction term

$$
V_{\mathrm{C}}\left(\mathbf{r}_{1}, \mathbf{r}_{2}, \mathbf{r}_{3}\right)=\sum_{i<j} V_{C}\left(r_{i j}\right)
$$

arising from the one-gluon exchange.

The EH depends explicitly on both bare quark masses $m_{i}$ and the constants $\mathrm{AF} \mu_{i}$ that finally acquire the meaning of the dynamical quark masses. As the first step the eigenvalue problem is solved for each set of $\mu_{i}$; then one has to minimize $\langle H\rangle$ with respect to $\mu_{i}$. Although being formally simpler the EH is equivalent to the relativistic Hamiltonian up to elimination of AF [14].

The formalism allows for a very transparent interpretation of $\mathrm{AF} \mu_{i}$ : starting from bare quark masses $m_{i}$, we arrive at the dynamical masses $\mu_{i}$ that appear due to the interaction and can be treated as the dynamical masses of constituent quarks. Therefore the constituent quark masses appear in the FCM calculations. These have obvious quark model analogs, but are derived directly using the AF formalism. Due to confinement $\mu_{i} \sim \sqrt{\sigma} \sim 400 \mathrm{MeV}$ or higher, even for the massless current quarks.

The baryon mass is given by

$$
\begin{gathered}
M_{B}=M_{0}+\Delta M_{\text {string }}+C, \\
M_{0}=\sum_{i=1}^{3}\left(\frac{m_{i}^{2}}{2 \mu_{i}}+\frac{\mu_{i}}{2}\right)+E_{0}\left(\mu_{i}\right)
\end{gathered}
$$

where $E_{0}\left(\mu_{i}\right)$ is an eigenvalue of the Schrödinger operator $H_{0}+V$, and the $\mu_{i}$ are defined by minimization condition

$$
\frac{\partial M_{0}\left(m_{i}, \mu_{i}\right)}{\partial \mu_{i}}=0
$$

\footnotetext{
${ }^{2}$ See Ref. [23]; for a brief review of the EH formalism relevant to the baryon problem considered in this paper see Sec. II of Ref. 11

${ }^{3}$ The spin-dependent effects for

baryons within the FCM will be considered elsewhere. For the

recent review of spin-dependent interactions in quarkonia in the framework the FCM see [24].
} 
The right-hand side of Eq. (4) contains the perturbative quark self-energy correction $C$ that is created by the color magnetic moment of a quark propagating through the vacuum background field [25]. This correction adds an overall negative constant to the hadron masses:

$$
C=-\frac{2 \sigma}{\pi} \sum_{i} \frac{\eta\left(t_{i}\right)}{\mu_{i}}, \quad t_{i}=m_{i} / T_{g}
$$

where $1 / T_{g}$ is the above mentioned gluonic correlation length. The numerical factor $\eta(t)$ arises from the evaluation of the integral

$$
\eta(t)=t \int_{0}^{\infty} z^{2} K_{1}(t z) e^{-z} d z
$$

where $K_{1}$ is the McDonald function. In what follows we use $T_{g}=1 \mathrm{GeV}$. Finally, $\Delta M_{\text {string }}$ in Eq. (4) is the so-called string correction to be discussed in the next section.

The above ingredients provide the basic mechanism that determines the baryon spectrum in the FCM.

\section{The string correction}

The string potential $V_{Y}\left(\mathbf{r}_{1}, \mathbf{r}_{2}, \mathbf{r}_{3}\right)$ in Eq. (2) represents only the leading term in the expansion of the QCD string Hamiltonian in terms of angular velocities [20. The leading correction in this expansion is known as a string correction. This is the correction totally missing in relativistic equations with local potentials. Its sign is negative, so the contribution of the string correction lowers the energy of the system, thus giving a negative contribution to the masses of orbitally excited states, leaving the $\mathrm{S}$-wave states intact.

As was mentioned in the introduction, so far, the string correction was calculated only for the orbitally excited heavy-light mesons [17] and hybrid charmonium states [21. For a baryon with the genuine string junction point the calculation of the string correction is a very cumbersome problem. The calculations are greatly simplified, however, if the string junction point is chosen to coincide with the center-of-mass coordinate $\boldsymbol{R}_{\mathrm{cm}}$. In this case, the complicated string junction potential is approximated by a sum of the one-body confining potentials. The accuracy of this approximation for the $P$-wave baryon states is better than $1 \%$ [14. Letting $\boldsymbol{R}_{c m}=0$ we arrive at the following form of the string potential

$$
V_{\text {string }}^{\mathrm{CM}}=\sigma \sum_{i}\left|\boldsymbol{r}_{i}\right| \int_{0}^{1} d \beta \sqrt{1-\boldsymbol{l}_{i}^{2}}
$$

where

$$
\boldsymbol{l}_{i}=\frac{\beta}{\left|\boldsymbol{r}_{i}\right|}\left[\boldsymbol{r}_{i} \times \dot{\boldsymbol{r}}_{i}\right]=\frac{\beta}{\mu_{i}\left|\boldsymbol{r}_{i}\right|}\left[\boldsymbol{r}_{i} \times \boldsymbol{p}_{i}\right]=-i \frac{\beta}{\mu_{i}\left|\boldsymbol{r}_{i}\right|}\left[\boldsymbol{r}_{i} \times \nabla_{i}\right] .
$$

Expanding the square roots in Eq. (9) in powers of the angular velocities $\boldsymbol{l}_{i}^{2}$ and keeping only the first two terms in this expansion, one obtains

$$
V_{\text {string }}^{\mathrm{CM}} \approx \sigma \sum_{i}\left|\boldsymbol{r}_{i}\right| \int_{0}^{1} d \beta\left(1-\frac{1}{2} \boldsymbol{l}_{i}^{2}\right)=\sigma \sum_{i}\left|\boldsymbol{r}_{i}\right|+\frac{\sigma}{6} \sum_{i}\left(\frac{1}{\mu_{i}^{2}\left|\boldsymbol{r}_{i}\right|}\left(\boldsymbol{r}_{i} \times \nabla_{i}\right)^{2}\right)
$$

and

$$
\Delta M_{\text {string }}=-\frac{\sigma}{6}<\Psi\left|\sum_{i} \frac{\left(\boldsymbol{r}_{i} \times \boldsymbol{p}_{i}\right)^{2}}{\mu_{i}^{2} r_{i}}\right| \Psi>,
$$

where $\Psi$ is an eigenfunction of the Hamiltonian (1). Further details and numerical results are given in Sec. 6.

\section{Coulomb-like interaction in the BPTh}

As was mentioned above, the spin-independent potential in Eq. (1) is the sum of the confinement potential and Coulomb-like interaction. Details of our treatment of the string junction confinement potential can be 
found in the appendix of Ref. [12. Let us now concentrate on the Coulomb-like part of interaction, $V_{C}(r)$. It is convenient to write the Coulomb-like potential in QCD in momentum space as

$$
V_{C}\left(\mathbf{q}^{2}\right)=-C_{F} \frac{\alpha_{V}\left(\mathbf{q}^{2}\right)}{\mathbf{q}^{2}}
$$

where $C_{F}$ is the color factor. In a baryon, the two quarks belong to the representation $\overline{\mathbf{3}}$ of $S U_{c}(3)$ for which $C_{F}=2 / 3$. A running constant $\alpha_{V}\left(\mathbf{q}^{2}\right)$ controls the behavior of standard perturbation theory (SPTh) at low momentum scales. The formal expression for $V_{C}(r)$ in position space can be written as

$$
V_{C}(r)=-C_{F} \frac{\alpha_{s}(r)}{r},
$$

where

$$
\alpha_{s}(r)=\frac{2}{\pi} \int_{0}^{\infty} d q \frac{\sin q r}{q} \alpha_{V}\left(\mathbf{q}^{2}\right)
$$

Up to two loops

$$
\alpha_{V}\left(\mathbf{q}^{2}\right)=\frac{4 \pi}{\beta_{0} t}\left(1-\frac{\beta_{1}}{\beta_{0}^{2}} \frac{\ln t}{t}\right)
$$

where $\beta_{i}$ are the coefficients of the QCD $\beta$-function,

$$
\beta_{0}=11-\frac{2}{3} n_{f}, \quad \beta_{1}=102-\frac{38}{3} n_{f},
$$

and

$$
t=\ln \frac{\mathbf{q}^{2}}{\Lambda_{V}^{2}}
$$

In what follows we use $n_{f}=3$.

The conventional coupling (16) is analytically singular at a scale $\mathbf{q}^{2}=\Lambda_{V}^{2}$, so the SPTh itself is not well defined in the infrared domain where the coupling become large. This problem can be traced back to the fact that the integral over the running coupling which appears in Eq.(15) is ill defined. As a result, $\alpha_{s}(r)$ is known only in the perturbative region, $r \lesssim 0.1 \mathrm{fm}$. Estimates of the average interquark distances in light baryons are in the vicinity of $0.7 \mathrm{fm}$ which is certainly outside of the perturbative region.

There also exists the possibility of defining a running coupling which stays finite in the infrared. One such example is the "timelike" effective coupling which is used in the dispersive approach 26. The idea is that such a coupling may give an effective measure of interaction at low scale [27. An alternative procedure is to define the fundamental coupling in QCD from a given physical observable [28. For our purposes, we find it convenient, as a useful approximation, to define the strong coupling constant $\alpha_{B}(r)$ in the BPTh [19]. In momentum space,

$$
\alpha_{B}\left(\mathbf{q}^{2}\right)=\alpha_{s}\left(\mathbf{q}^{2}+m_{B}^{2}\right)
$$

where $m_{B} \sim 1 \mathrm{Gev}$ is the appropriate mass parameter 4 . The logic behind Eq. (19) is that the perturbative gluon propagator is modified strongly at $q \lesssim m_{B}$ by the physics of large distances. This result can be conventionally viewed as arising from the interaction of a gluon with background vacuum fields.

We define $\alpha_{B}(r)$, as well as the Coulomb-like potential in the configuration space via expressions similar to (14) and (15)

$$
\begin{gathered}
\alpha_{B}(r)=\frac{2}{\pi} \int_{0}^{\infty} d q \frac{\sin q r}{q} \alpha_{B}\left(\mathbf{q}^{2}\right), \\
V_{C}(r)=-C_{F} \frac{\alpha_{B}(r)}{r} .
\end{gathered}
$$

For $\alpha_{B}\left(\mathbf{q}^{2}\right)$, we use the two-loop result (16) with the substitution

$$
t \rightarrow t_{B}=\ln \frac{\mathbf{q}^{2}+m_{B}^{2}}{\Lambda_{V}^{2}}
$$

\footnotetext{
4 This parameter has the meaning of being the lowest hybrid excitation, $m_{B}=M(Q \bar{Q} g g)-M(Q \bar{Q} g)$; from comparison with the lattice static potential $m_{B} \sim 1 \mathrm{GeV}$.
} 
The resulting coupling $\alpha_{B}\left(\mathbf{q}^{2}\right)$ is finite in the infrared, $\mathbf{q}^{2} \rightarrow 0$. In the ultraviolet region, $\mathbf{q}^{2} \gg m_{B}^{2}$ one recovers the SPTh result. In configuration space the background coupling constant $\alpha_{B}(r)$ exists for all distances and saturates at some critical, or freezing, value for $r \gg 1 / m_{B}$. The specific choice of the parameters in Eq. (22) will be discussed in Sec. 6.

\section{Hyperspherical formalism for three-quark systems.}

Following our previous analysis [12] we use the hyperspherical method to calculate the masses of the ground and excited hyperon states. The idea of hyperspherical method is to generalize the simplicity of the spherical harmonic expansion for the angular functions of a two particle in three dimensional space to a system of $\mathrm{N}$ particles by introducing in a 3N-3 dimensional space a global length $R$ called the hyperradius, and a set of angles, $\Omega$. In this section, we briefly review the hyperspherical formalism as applied to our specific problem.

Let us introduce in the system of three quarks with dynamical masses $\mu_{i}$ and coordinates $\boldsymbol{r}_{i}$ the threebody Jacobi coordinates in the six-dimensional coordinate space as

$$
\boldsymbol{\rho}_{i j}=\sqrt{\frac{\mu_{i j}}{\mu_{0}}}\left(\boldsymbol{r}_{i}-\boldsymbol{r}_{j}\right), \quad \lambda_{i j}=\sqrt{\frac{\mu_{i j, k}}{\mu_{0}}}\left(\frac{\mu_{i} \boldsymbol{r}_{i}+\mu_{j} \boldsymbol{r}_{j}}{\mu_{i}+\mu_{j}}-\boldsymbol{r}_{k}\right),
$$

$\left(i, j, k\right.$ cyclic), where $\mu_{i j}$ and $\mu_{i j, k}$ are the reduced dynamical quark masses:

$$
\mu_{i j}=\frac{\mu_{i} \mu_{j}}{\mu_{i}+\mu_{j}}, \quad \mu_{i j, k}=\frac{\left(\mu_{i}+\mu_{j}\right) \mu_{k}}{\mu_{i}+\mu_{j}+\mu_{k}},
$$

and $\mu_{0}$ is an arbitrary parameter with the dimension of mass, which drops out in the final expressions. The baryon wave function depends on the Jacobi coordinates (23). Now we switch from Jacobi coordinates to hyperspherical coordinates in coordinate space

$$
\begin{aligned}
& R^{2}=\rho^{2}+\lambda^{2}, \\
& \rho=R \sin \theta, \quad \lambda=R \cos \theta, \quad 0 \leq \theta \leq \pi / 2,
\end{aligned}
$$

where $R$ is the six-dimensional hyperradius that is invariant under quark permutations. In what follows we omit the indices $i$ and $j$.

The explicit expression for the kinetic energy operator $H_{0}$ for three-quark systems in the center-of-mass system is remarkably simple in hyperspherical coordinates

$$
H_{0}=-\frac{1}{2 \mu_{0}}\left(\frac{\partial^{2}}{\partial R^{2}}+\frac{5}{R} \frac{\partial}{\partial R}+\frac{\boldsymbol{L}^{2}(\Omega)}{R^{2}}\right) .
$$

In Eq. (26) $\Omega$ denotes the five angular coordinates $\theta, \boldsymbol{n}_{\rho}, \boldsymbol{n}_{\lambda}$, and $\boldsymbol{L}^{2}(\Omega)$ is a squared hyperangular momentum operator. Its eigenfunctions (the hyperspherical harmonics) are defined by

$$
\mathbf{L}^{2}(\Omega) Y_{[K]}\left(\theta, \mathbf{n}_{\rho}, \mathbf{n}_{\lambda}\right)=-K(K+4) Y_{[K]}\left(\theta, \mathbf{n}_{\rho}, \mathbf{n}_{\lambda}\right),
$$

with $K$ being the grand orbital momentum.

The wave function $\psi(\boldsymbol{\rho}, \boldsymbol{\lambda})$ is written in a symbolic shorthand notation as

$$
\psi(\boldsymbol{\rho}, \boldsymbol{\lambda})=\sum_{[K]} \psi_{[K]}(R) Y_{[K]}(\Omega),
$$

where the set $[K]$ is defined by the orbital momentum of the state and the symmetry properties.

In what follows we use the ansatz $K=K_{\min }$, where $K_{\min }=0$ for $L=0$ and $K_{\min }=1$ for $L=1$. The accuracy of this approximation has been discussed in [14. Our task is then extremely simple in

principle: we have to choose a zero-order wave function corresponding to the minimal $K$ for a given $L$. The corresponding hyperspherical harmonics are

$$
\begin{aligned}
& Y_{0}=\sqrt{\frac{1}{\pi^{3}}}, \quad K=0, \\
& \boldsymbol{Y}_{\rho}=\sqrt{\frac{6}{\pi^{3}}} \frac{\boldsymbol{\rho}}{R}, \quad \boldsymbol{Y}_{\lambda}=\sqrt{\frac{6}{\pi^{3}}} \frac{\lambda}{R}, \quad K=1 .
\end{aligned}
$$


For nns baryons we use the basis in which the strange quark is singled out as quark 3 but in which the nonstrange quarks are still antisymmetrized. In the same way, for the ssn baryon we use the basis in which the nonstrange quark is singled out as quark 3. The nns basis states diagonalize the confinement problem with eigenfunctions that correspond to separate excitations of the nonstrange and strange quarks ( $\rho$ - and $\lambda$ excitations, respectively). In particular, excitation of the $\boldsymbol{\lambda}$ variable unlike excitation in $\boldsymbol{\rho}$ involves the excitation of the "odd" quark ( $s$ for $n n s$ or $n$ for $s s n$ ). The nonsymmetrized $u d s$ and $s s q$ bases usually provide a much simplified picture of the states 33 .

For the purpose of implementing our truncation scheme, we introduce the reduced functions $u_{\nu}(R)$ :

$$
\Psi_{\nu}(R, \Omega)=\frac{u_{\nu}(R)}{R^{5 / 2}} \cdot Y_{\nu}(\Omega),
$$

where $\nu=0$ for $L=0$, and $\nu=\rho, \lambda$ for $L=15$, as well as a new variable

$$
x=\sqrt{\mu_{0}} R=\left(\sum_{i} \frac{\mu_{1} \mu_{2}}{M} r_{12}^{2}+\frac{\mu_{2} \mu_{3}}{M} r_{23}^{2}+\frac{\mu_{3} \mu_{1}}{M} r_{31}^{2}\right)^{1 / 2},
$$

that does not depend on $\mu_{o}$. Inserting Eq. (30) into the Schrödinger equation for $\Psi_{\nu}(R, \Omega)$ and averaging the interaction $V=V_{Y}+V_{C}$ over the six-dimensional sphere $\Omega$ with the weight $\left|Y_{\nu}\right|^{2}$, one obtains the one-dimensional Schrödinger equation for $u_{\nu}(x)$

$$
\frac{d^{2} u_{\nu}(x)}{d x^{2}}+2\left(E_{0}-\frac{\left(K+\frac{3}{2}\right)\left(K+\frac{5}{2}\right)}{2 x^{2}}-V_{\mathrm{Y}}^{\nu}(x)-V_{\mathrm{C}}^{\nu}(x)\right) u_{\nu}(x)=0,
$$

where

$$
V_{\mathrm{Y}}^{\nu}(x)=\int\left|Y_{\nu}(\theta, \chi)\right|^{2} V_{\mathrm{Y}}\left(\mathbf{r}_{1}, \mathbf{r}_{2}, \mathbf{r}_{3}\right) d \Omega=\sigma b_{\nu} x
$$

and

$$
V_{\text {Coulomb }}^{\nu}(x)=-\frac{2}{3} \int\left|Y_{\nu}(\theta, \chi)\right|^{2} \sum_{i<j} \frac{\alpha_{B}\left(r_{i j}\right)}{r_{i j}} d \Omega=-\frac{2}{3} \frac{a_{B}^{\nu}(x)}{x} .
$$

The constants $b_{\nu}$ in Eq. (33) are defined by the two-dimensional integrals in the plane $(\theta, \cos \varphi=\hat{\rho} \hat{\boldsymbol{\lambda}})$. Explicit expressions for these integrals are written in the appendix of Ref. [12. The functions $a_{B}^{\nu}(x)$ are

$$
a_{B}^{\nu}(x)=\sum_{i<j} \sqrt{\mu_{i j}} \int \alpha_{B}\left(\frac{x \sin \theta}{\sqrt{\mu_{i j}}}\right) \frac{d \omega_{\nu}}{\sin \theta},
$$

where

$$
d \omega_{0}=\frac{16}{\pi} \sin ^{2} \theta \cos ^{2} \theta d \theta
$$

and

$$
d \omega_{\rho}=\frac{32}{\pi} \sin ^{4} \theta \cos ^{2} \theta d \theta, \quad d \omega_{\lambda}=\frac{32}{\pi} \sin ^{2} \theta \cos ^{4} \theta d \theta .
$$

\section{Results and discussion}

In the previous sections, we introduced the Hamiltonian we use to obtain baryon spectra. This Hamiltonian contains five parameters: the current quark masses $m_{n}$ and $m_{s}$, the string tension $\sigma$, and two parameters $\Lambda_{V}$ and $m_{B}$ defining $\alpha_{B}\left(\mathbf{q}^{2}\right)$ in Eq. (22). Let us underline that they are not the fitting parameters. In our calculations we use $\sigma=0.15 \mathrm{GeV}^{2}$ found in the $\mathrm{SU}(3)$ QCD lattice simulations [29]. We employ the current light quark masses $m_{u}=m_{d}=7 \mathrm{MeV}$ and the bare strange quark mass $m_{s}=175 \mathrm{MeV}$ found previously from the fit to $D_{s}$ spectra [17. This value of the strange quark mass is consistent with the QCD sum rules estimation $m_{s}(2 \mathrm{GeV})=(125 \pm 40) \mathrm{MeV}$ [30]. More recent estimation yields

\footnotetext{
${ }^{5}$ In what follows, for ease of notation we will drop the magnetic quantum numbers of the vector spherical harmonics.
} 


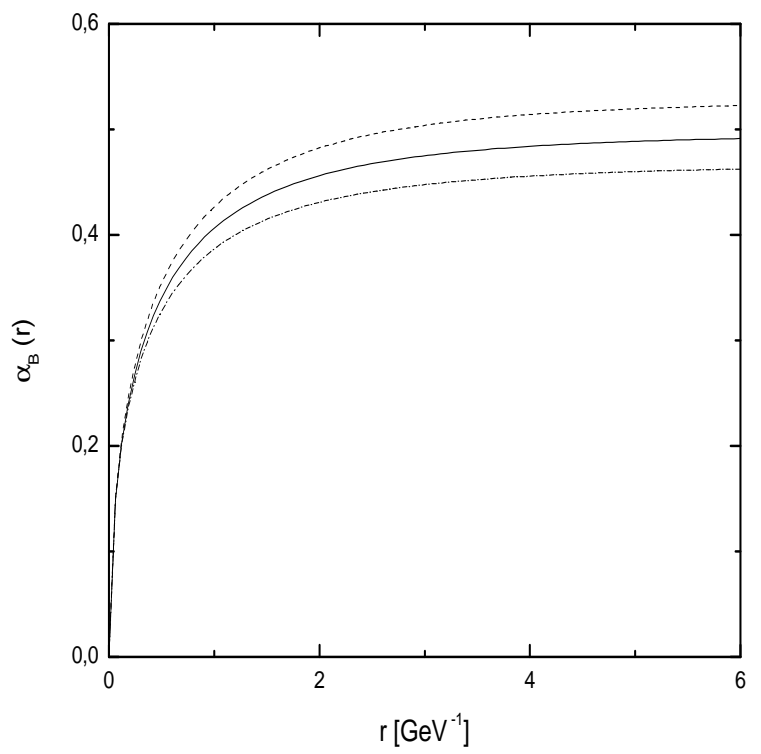

Figure 1: The dependence of the running coupling constant $\alpha_{B}(r)$ on the distance for $m_{B}=1 \mathrm{GeV}$ and $\Lambda_{V}=0.36$ (solid line), 0.38 (dashed line) and $0.34 \mathrm{GeV}$ (dotted-dashed line)

Table 1: The freezing values of $\alpha_{B}(\infty)$ for the different choices of $\Lambda_{V}$ and $m_{B}$. The values of $\Lambda_{V}$ and $m_{B}$ are given in $\mathrm{GeV}$.

\begin{tabular}{|c|c|c|c|c|c|c|c|c|c|}
\hline$\Lambda_{V}$ & & 0.34 & & & 0.36 & & & 0.38 & \\
\hline$m_{B}$ & 0.95 & 1.00 & 1.05 & 0.95 & 1.00 & 1.05 & 0.95 & 1.00 & 1.05 \\
\hline$\alpha_{B}(\infty)$ & 0.492 & 0.467 & 0.445 & 0.526 & 0.496 & 0.471 & 0.563 & 0.528 & 0.500 \\
\hline
\end{tabular}

$m_{s}(2 \mathrm{GeV})=(90 \pm 10) \mathrm{MeV}$ 31. Recall that in our Hamiltonian approach the current mass $m_{s}$ enters at a much lower scale. For the remaining two parameters $\Lambda_{V}$ and $m_{B}$ in Eq. (22) we employ the values

$$
\Lambda_{V}=(0.36 \pm 0.02) \mathrm{GeV}, \quad m_{B}=(1 \pm 0.05) \mathrm{GeV},
$$

determined previously in Ref. [32. The result is consistent with the freezing of $\alpha_{B}(r)$ with a magnitude $\sim 0.5-0.6$, see Table 1 The behavior of $\alpha_{B}(r)$ for $\Lambda_{V}=0.36 \mathrm{GeV}$ is shown in Fig. 1 for three different values of $m_{B}$. We use the error bars in Eq. (38) to illustrate the sensitivity of the baryon masses to the chosen input. Note that $\alpha_{B}(r)$ increases with $\Lambda_{V}$ and, for fixed $\Lambda_{V}$, decreases with $m_{B}$.

We begin the discussion of our results by examining the predictions for the ground states of the nnn, nns and snn baryon with $L=0$. In Table 2 are displayed the $n n n, n n s$ and snn masses for the three choices of $\Lambda_{V}: 0.34,0.36$ and $0.38 \mathrm{GeV}$, and three choices of $m_{B}: 0.95,1.00$ and $1.05 \mathrm{GeV}$. In this table we also show the dynamical masses $\mu_{i}$ found from the minimum condition (6).

The results in Table 2 show that the baryon masses decrease when $\Lambda_{V}$ increases and, for fixed $\Lambda_{V}$, the baryon masses decrease when $m_{B}$ increases. This comes as no surprise: the effect can be easily read off from the results of Table 1 and Fig. 1. Increasing $\Lambda_{V}$ for fixed $m_{B}$ and decreasing $m_{B}$ for fixed $\Lambda_{V}$ leads to increased running constant $\alpha_{B}(r)$ and respectively, to decreased baryon masses. Upon varying the parameters $\Lambda_{V}$ and $m_{B}$, we obtain the baryon masses in the interval 1161-1209 $\mathrm{MeV}(\mathrm{nnn}), 1246-1297$ $\mathrm{MeV}(n n s)$, and $1330-1383 \mathrm{MeV}(\mathrm{ssn})$. The difference in the mass values is mostly due to the difference in the running of the strong coupling in the midmomentum regime.

It is instructive to compare the results obtained for the running coupling constant (RCC) with those 
Table 2: The masses of the nnn, nns and ssn baryons with $L=0$. All quantities are given in MeV.

\begin{tabular}{|c|c|c|c|c|c|c|c|c|c|c|}
\hline & & & nnn & & & nns & & & $s s n$ & \\
\hline$\Lambda_{V}$ & $m_{B}$ & $\mu_{1}$ & $\mu_{3}$ & $M_{B}$ & $\mu_{1}$ & $\mu_{3}$ & $M_{B}$ & $\mu_{1}$ & $\mu_{3}$ & $M_{B}$ \\
\hline \multirow[t]{3}{*}{340} & 1050 & 407 & 407 & 1209 & 412 & 452 & 1297 & 457 & 417 & 1383 \\
\hline & 1000 & 409 & 409 & 1201 & 414 & 453 & 1288 & 458 & 419 & 1373 \\
\hline & 950 & 410 & 410 & 1190 & 415 & 455 & 1277 & 460 & 421 & 1363 \\
\hline \multirow[t]{3}{*}{360} & 1050 & 409 & 409 & 1197 & 415 & 454 & 1286 & 459 & 420 & 1371 \\
\hline & 1000 & 411 & 411 & 1187 & 417 & 456 & 1276 & 461 & 422 & 1360 \\
\hline & 950 & 414 & 414 & 1177 & 419 & 458 & 1263 & 463 & 424 & 1347 \\
\hline \multirow[t]{3}{*}{380} & 1050 & 412 & 412 & 1186 & 418 & 457 & 1274 & 462 & 423 & 1358 \\
\hline & 1000 & 414 & 414 & 1174 & 420 & 459 & 1252 & 464 & 425 & 1345 \\
\hline & 950 & 417 & 417 & 1161 & 422 & 461 & 1246 & 466 & 428 & 1330 \\
\hline
\end{tabular}

obtained for the freezing coupling constant $(\mathrm{FCC}) \alpha_{s}^{(0)}=0.39$ 12. In Table 3 such comparison is presented for the $S$ - and $P$-baryon states for the particular set of the parameters $\Lambda_{V}=(0.36 \pm 0.02) \mathrm{GeV}$ and $m_{B}=1 \mathrm{GeV}$. In this table we compare the dynamical masses $\mu_{i}$ and the baryon masses $M_{0}+C$ in Eq. (4). The quantities shown in the rows labeled by RCC have been calculated for $m_{B}=1.00 \mathrm{GeV}$, the central results correspond to $\Lambda_{V}=0.36 \mathrm{GeV}$, the upper error bars correspond to $\Lambda_{V}=0.34 \mathrm{GeV}$, and the lower error bars correspond to $\Lambda_{V}=0.38 \mathrm{GeV}$.

From Table 3, it can be seen that for the $S$-states the baryon masses calculated for the RCC and FCC are all in agreement with each other within the error bars, although the central values for the nnn, nns and $s s n$ states are typically lower by about $20 \mathrm{MeV}$ than those for the FCC. For the $P$-waves, the central values of the masses of $\rho$ excitations are typically $20 \mathrm{MeV}$ heavier than those for the FCC while the central mass values of the $\lambda$ excitations are smaller by about $50 \mathrm{MeV}$ than those values calculated for $\alpha_{s}^{(0)}=0.39$. Note that introducing the RCC eliminates the degeneracy of the $\rho$ and $\lambda$-excitations for the $n n n$ states found previously for the FCC [14.

Now let us consider the string correction for the confinement potential for the orbital excitations. Explicit calculation [34] of the matrix elements in Eq. (12) yields

$$
\begin{gathered}
\Delta M_{\text {string }}^{\rho}=-\frac{64 \sigma}{45 \pi} \cdot \frac{1}{\mu^{3 / 2}} \sqrt{\frac{1+\kappa}{2+\kappa}} \gamma_{\rho}, \\
\Delta M_{\text {string }}^{\lambda}=-\frac{64 \sigma}{45 \pi} \cdot \frac{\kappa}{\mu^{3 / 2}(2+\kappa)^{3 / 2}}\left(\frac{1}{\kappa^{5 / 2}}+2 \sqrt{1+\kappa}\right) \gamma_{\lambda},
\end{gathered}
$$

where $\mu_{1}=\mu_{2}=\mu, \kappa=\mu_{3} / \mu$,

$$
\gamma_{\nu}=\int_{0}^{\infty} \frac{u_{\nu}^{2}(x)}{x} d x, \quad \nu=\rho, \lambda,
$$

and the wave functions $u_{\nu}(x)$ are normalized to unity.

The wave function corrections $\gamma_{\nu}$ in (41) which influence the string correction does not essentially depend on the baryon flavor nor on the type of excitation. E.g. for $\Lambda_{V}=0.36 \mathrm{GeV}$ and $m_{B}=1 \mathrm{GeV}$ we 
Table 3: Comparison of the baryon masses $M_{0}+C$ in Eq. (44) calculated using the RCC $\alpha_{B}(r)$ without taking into account the string correction with those obtained for the FCC $\alpha_{s}=0.39$ from Ref. 14. The quantities shown in the rows labeled by RCC have been calculated for $\Lambda_{V}=360 \mathrm{MeV}, m_{B}=1000 \mathrm{MeV}$, the upper error bars correspond to $\Lambda_{V}=340 \mathrm{MeV}$, and the lower error bars correspond to $\Lambda_{V}=380$ $\mathrm{MeV}$. The masses of the baryons and dynamical masses $\mu_{i}$ are given in $\mathrm{MeV}$.

\begin{tabular}{|c|c|c|c|c|c|c|c|c|c|c|}
\hline & \multicolumn{4}{|c|}{$n n n$} & \multicolumn{3}{|c|}{$n n s$} & \multicolumn{3}{|c|}{ ssn } \\
\hline & $\mathbf{L}$ & $\mu_{1}$ & $\mu_{3}$ & $M_{0}+C$ & $\mu_{1}$ & $\mu_{3}$ & $M_{0}+C$ & $\mu_{1}$ & $\mu_{3}$ & $M_{0}+C$ \\
\hline $\mathrm{RCC}$ & 0 & $411_{-4}^{+6}$ & $411_{-4}^{+6}$ & $1187_{-26}^{+22}$ & $417_{-5}^{+5}$ & $456_{-4}^{+5}$ & $1276_{-30}^{+22}$ & $461_{-5}^{+5}$ & $422_{-5}^{+6}$ & $1360_{-30}^{+23}$ \\
\hline FCC & & 408 & 408 & 1209 & 414 & 453 & 1298 & 458 & 419 & 1384 \\
\hline $\mathrm{RCC}$ & $\mathbf{1}_{\rho}$ & $454_{-2}^{+2}$ & $454_{-2}^{+2}$ & $1695_{-10}^{+9}$ & $477_{-2}^{+2}$ & $460_{-2}^{+2}$ & $1774_{-10}^{+9}$ & $516_{-2}^{+4}$ & $424_{-2}^{+3}$ & $1832_{-10}^{+9}$ \\
\hline FCC & & 457 & 457 & 1674 & 482 & 459 & 1751 & 520 & 424 & 1810 \\
\hline $\mathrm{RCC}$ & $\mathbf{1}_{\lambda}$ & $469_{-3}^{+4}$ & $469_{-3}^{+4}$ & $1625_{-15}^{+13}$ & $456_{-4}^{+2}$ & $540_{-3}^{+2}$ & $1687_{-15}^{+13}$ & $496_{-3}^{+3}$ & $513_{-3}^{+3}$ & $1771_{-15}^{+14}$ \\
\hline FCC & & 457 & 457 & 1674 & 441 & 534 & 1738 & 483 & 506 & 1827 \\
\hline
\end{tabular}

get (in units of $\mathrm{GeV}^{1 / 2}$ )

$$
\gamma_{\rho}=0.328(n n n), \quad \gamma_{\rho}=0.326(n n s), \quad \gamma_{\rho}=0.325(\text { ssn }),
$$

and

$$
\gamma_{\lambda}=0.333(\text { nnn }), \quad \gamma_{\lambda}=0.332(\text { nns }), \quad \gamma_{\lambda}=0.331(\text { ssn }) .
$$

The results for the $P$-wave excitations of the $n n n, n n s$ and $s s n$ baryons when are taking into account the string correction along with the values for the string correction itself are shown in Table 4. Analysis of the data shows that string correction does not essentially depend on the baryon flavor nor on the type of excitation. As a result of the weak dependence of the string correction on a baryon flavor and the type of excitation the masses for all considered baryons become smaller by about the same value $\sim 50-60 \mathrm{MeV}$ (see the $6^{\text {th }}$ column of Table 4).

The baryon energies agree reasonably well with the Particle Data Group (PDG) listing particularly if we take into consideration that spin interactions are neglected. For instance, for $L=0$ we get $\frac{1}{4}(\Lambda+$ $\left.\Sigma+2 \Sigma^{*}\right)_{\text {theory }}=1276_{-30}^{+23} \mathrm{MeV}$, where the error bars correspond to the variation of the hyperon masses within the chosen range of $\Lambda_{V}$ and $m_{B}$ versus $\frac{1}{4}\left(\Lambda+\Sigma+2 \Sigma^{*}\right)_{\exp }=1267 \mathrm{MeV}$. For the $\Xi$, we have $\Xi_{\text {theory }}=1360_{-30}^{+23} \mathrm{MeV}$, whereas $\Xi_{\exp }=1315 \mathrm{MeV}$. However, for the nucleon we get $\frac{1}{2}(N+\Delta)_{\text {theory }}=$ $1187_{-21}^{+22} \mathrm{MeV}$, which is about $100 \mathrm{MeV}$ heavier than $\frac{1}{2}(N+\Delta)_{\exp }=1085 \mathrm{MeV}$. The difference can be ascribed to the effects of spin-dependent quark-quark interactions modeled after the effect of gluon exchange in QCD [35] or arising from one-boson exchange [36] that are completely omitted in the present approach 6. Another source of the discrepancy is the systematic error associated with the use of the AF formalism, which is maximal for the $S$-wave $n n n$ states [14.

\footnotetext{
${ }^{6}$ Note that the one-boson exchange effects are important for baryons containing the scalar $n n$ diquarks $(N$ and $\Lambda)$ and much less important for the baryons containing the axial diquarks $(\Delta$ and $\Sigma)$, see e.g. Ref. 37.
} 
Table 4: Masses of the $\rho$ and $\lambda$ baryon excitations for $m_{B}=1000 \mathrm{MeV}$ taking into account the string corrections, Eqs. (39), (40). The masses of the baryons, string corrections and dynamical masses $\mu_{i}$ are given in $\mathrm{MeV}$.

\begin{tabular}{|c|c|c|c|c|c|c|c|}
\hline Baryon & Excitation & $\Lambda_{V}$ & $\mu_{1}=\mu_{2}$ & $\mu_{3}$ & $M_{0}+C$ & $\Delta M_{\text {string }}$ & $M_{B}$ \\
\hline nnn & $\mathbf{1}_{\rho}$ & $\begin{array}{l}340 \\
360 \\
380\end{array}$ & $\begin{array}{l}452 \\
454 \\
456\end{array}$ & $\begin{array}{l}452 \\
454 \\
456\end{array}$ & $\begin{array}{l}1704 \\
1695 \\
1685\end{array}$ & $\begin{array}{l}-60 \\
-59 \\
-59\end{array}$ & $\begin{array}{l}1644 \\
1636 \\
1626\end{array}$ \\
\hline & $\mathbf{1}_{\lambda}$ & $\begin{array}{l}340 \\
360 \\
380\end{array}$ & $\begin{array}{l}466 \\
469 \\
471\end{array}$ & $\begin{array}{l}466 \\
469 \\
471\end{array}$ & $\begin{array}{l}1638 \\
1625 \\
1610\end{array}$ & $\begin{array}{l}-58 \\
-58 \\
-57\end{array}$ & $\begin{array}{l}1580 \\
1567 \\
1553\end{array}$ \\
\hline nns & $\mathbf{1}_{\rho}$ & $\begin{array}{l}340 \\
360 \\
380\end{array}$ & $\begin{array}{l}475 \\
477 \\
479\end{array}$ & $\begin{array}{l}458 \\
460 \\
462\end{array}$ & $\begin{array}{l}1783 \\
1774 \\
1764\end{array}$ & $\begin{array}{l}-55 \\
-55 \\
-55\end{array}$ & $\begin{array}{l}1728 \\
1719 \\
1709\end{array}$ \\
\hline nns & $\mathbf{1}_{\lambda}$ & $\begin{array}{l}340 \\
360 \\
380\end{array}$ & $\begin{array}{l}452 \\
455 \\
458\end{array}$ & $\begin{array}{l}537 \\
540 \\
542\end{array}$ & $\begin{array}{l}1700 \\
1687 \\
1672\end{array}$ & $\begin{array}{l}-51 \\
-51 \\
-51\end{array}$ & $\begin{array}{l}1649 \\
1636 \\
1621\end{array}$ \\
\hline$s s q$ & $\mathbf{1}_{\rho}$ & $\begin{array}{l}0.34 \\
0.36 \\
0.38\end{array}$ & $\begin{array}{l}514 \\
516 \\
518\end{array}$ & $\begin{array}{l}422 \\
424 \\
427\end{array}$ & $\begin{array}{l}1841 \\
1832 \\
1822\end{array}$ & $\begin{array}{l}-48 \\
-48 \\
-48\end{array}$ & $\begin{array}{l}1793 \\
1784 \\
1774\end{array}$ \\
\hline & $\mathbf{1}_{\lambda}$ & $\begin{array}{l}0.34 \\
0.36 \\
0.38\end{array}$ & $\begin{array}{l}493 \\
496 \\
499\end{array}$ & $\begin{array}{l}510 \\
513 \\
516\end{array}$ & $\begin{array}{l}1785 \\
1771 \\
1756\end{array}$ & $\begin{array}{l}-51 \\
-51 \\
-51\end{array}$ & $\begin{array}{l}1734 \\
1720 \\
1705\end{array}$ \\
\hline
\end{tabular}

The physical $P$-wave states are not pure $\rho$ or $\lambda$ excitations but linear combinations of all states with a given total momentum $J$. Most physical states are, however, close to pure $\rho$ or $\lambda$ states 33. For example, the masses of $\mathrm{N}(1535)$ and $\mathrm{N}(1520)$ resonances with $J^{P}=\frac{1}{2}^{-}$and $J^{P}=\frac{3}{2}^{-}$, respectively, both match with the mass of the $\lambda$-excitation for the $n n n$ baryon from Table $4 M_{\lambda}(n n n)=1567_{-14}^{+13} \mathrm{MeV}$. The masses of $\Sigma(1620)$ and $\Sigma(1670)$ states with $J^{P}=\frac{1}{2}^{-}$and $J^{P}=\frac{3}{2}^{-}$, respectively, match very closely with the mass of the $\lambda$-excitation for the nns baryon: $M_{\lambda}(n n s)=1636_{-15}^{+13} \mathrm{MeV}$. The masses of the $\rho$-excitations which correspond to $P$-states of the light diquarks are typically $60-80 \mathrm{MeV}$ higher.

The comparison of our result for the negative parity ground state in the $\Xi$ channel, $M_{\lambda}(s s n)=1720_{-15}^{+14}$ $\mathrm{MeV}$ with the other theoretical predictions for this state and the result from the PDG listing is presented in Table 5 . 
Table 5: Low-lying $\Xi$ spectrum of spin $L=1$ predicted by the nonrelativistic quark models of Chao, Isgur and Karl 33 and of Pervin and Roberts 38, the relativized quark model of Capstick and Isgur 39, the Glozman-Riska model [36], the large $N_{c}$ analysis [6], the algebraic model [5], QCD sum rules [40, and the Skyrme model [4]. The question mark in the last column means that the $J^{P}$ quantum numbers are not identified by PDG. The masses are given in MeV.

\begin{tabular}{l|cccccccccc}
\hline \hline State & {$[33]$} & {$[38]$} & {$[39$} & {$[36$} & {$[6]$} & {$[5]$} & {$[40]$} & {$[4]$} & This work & PDG \\
\hline & & & & & & & & & & \\
$\Xi\left(\frac{1}{2}^{-}\right)$ & 1785 & 1725 & 1755 & 1758 & 1780 & 1869 & 1550 & 1660 & $1720_{-15}^{+14}$ & $\Xi(1690) ?$ \\
$\Xi\left(\frac{3}{2}^{-}\right)$ & 1800 & 1759 & 1785 & 1758 & 1815 & 1828 & 1840 & 1820 & $1720_{-15}^{+14}$ & $\Xi(1820)$ \\
\hline \hline
\end{tabular}

\section{Conclusions}

In this paper the study of the ground and excited states of the $q q q, q q s$ and $s s q$ baryons by including into the EH derived using the FCM the effects of the running strong coupling constant $\alpha_{B}\left(r_{i j}\right)$ in the perturbative Coulomb-like part of the three-quark potential is presented. The results have refined our previous studies obtained for the freezing coupling constant. The three-quark problem has been solved using the hyperspherical approach. The string correction for the confinement potential of the orbitally excited baryons is estimated. For each baryon, we have calculated the dynamical quark masses $\mu_{i}$ from Eq. (6) and the baryon masses (4) with the self-energy corrections (7) and the string corrections (39), (40). The main results are given in Tables 2 - 4. Our study shows that a fairly good description of the $S$ and $P$-wave baryons can be obtained with spin independent energy eigenvalues corresponding to the confining along with Coulomb potentials. We emphasize that no fitting parameter were used in our calculations. This comparative study provides deeper insight into the quark model results for which the constituent masses encode the QCD dynamics.

This work was supported in part by RFBR Grants No. 06-02-17120, No. 08-02-00657, No. 08-02-00677, and by the grant for scientific schools NSh.4961.2008.2 .

\section{References}

[1] A recent overview of quark models is given in C. Amsler, T. DeGrand and B. Krusche, Quark Models, in the review section of PDG'08, C. Amsler et al., Phys. Lett. B 667, 1 (2008).

[2] E. Bagan et al., Z. Phys. C64, 37 (1994); see also D. W. Wang and M. Q. Huang, Phys. Rev. D68, 034019 (2003) and references therein

[3] A. F. Falk, Phys. Rev. Lett. 77, 223 (1996); E. F. Jenkins, Phys. Rev. D54, 4515 (1996), Phys. Rev. D55, 10 (1997)

[4] Yongseok Oh, Phys. Rev. D75, 074002 (2007)

[5] R. Bijker, F. Iachello, and A. Leviatan, Ann. Phys. (N.Y.) 284, 89 (2000).

[6] C. E. Carlson and C. D. Carone, Phys. Lett. B 484, 260 (2000); C. L. Schat, J. L. Goity, and N. N. Scoccola, Phys. Rev. Lett. 88, 102002 (2002); Phys. Lett. B 564, 83 (2003); N. Matagne and Fl. Stancu, Phys. Rev. D 71, 014010 (2005); Phys. Rev. D 74, 034014 (2006); C. Semay, F. Buisseret, N. Matagne, and Fl. Stancu, Phys. Rev. D 75, 096001 (2007).

[7] C. Aubin et al. [MILC Collaboration], Phys. Rev. D70, 094505 (2004); C. T. H. Davys et al. [HP QCD Collaboration], Phys. Rev. Lett. 92, 022001 (2004); M. Wingate et al., Phys. Rev. Lett. 92, 160001 (2004) 
[8] Huey-Wen Lin it et al. e-print arXiv: hep-lat/0810.3588 and references therein.

[9] T. Burch et al., Phys. Rev. D 74, 014504 (2006)

[10] H. G. Dosch, Phys. Lett. 190, (1987) 177, H. G. Dosch, Yu. A. Simonov, Phys. Lett. 202, 339 (1988); Yu. A. Simonov, Nucl. Phys. B307, 512 (1988); for a review see A. Di Giacomo, H. G. Dosch, V. I. Shevchenko, Yu. A. Simonov, Phys. Rep. 372319 (2002).

[11] I. M. Narodetskii and M. A. Trusov, Phys. Atom. Nucl. 67, 762 (2004) [Yad. Fiz. 67, 783 (2004)].

[12] O. N. Driga, I. M. Narodetskii, A. I. Veselov, Phys. Atom. Nucl. 71, 335 (2008) [Yad. Fiz. 71, 356 (2008)], e-print ArXiv: hep-ph/0712.1479.

[13] I. M. Narodetskii, M. A. Trusov and A. I. Veselov, Phys. Atom. Nucl. (in press, March 2009), e-print arXiv: hep-ph/0801.1980; I. L. Grach, I. M. Narodetskii, M .A .Trusov and A. I. Veselov, e-print arXiv: hep-ph/0811.2184.

[14] I. M. Narodetskii, C. Semay, A. I. Veselov, Eur. Phys. J. C 55, 403 (2008).

[15] A. M. Badalyan and B. L. G. Bakker, Phys. Rev. D 66034025 (2002), Phys. Rev. D 70, 016007 (2004).

[16] E. Eichten et al., Phys. Rev. Lett. 34, 369 (1975), Phys. Rev. D 21, 203 (1980).

[17] Yu. S. Kalashnikova, A. Nefediev, Yu. A. Simonov, Phys, Rev. D 64, 014037 (2001).

[18] see e.g. M. Jezabek, M. Peter, and Y. Sumino, Phys. Lett. B428, 352 (1998).

[19] Yu. A. Simonov, Phys. Atom. Nucl. 58, 107 (1995), [Yad. Fiz. 58, 113 (1995)].

[20] A. Yu. Dubin, A. B. Kaidalov and Yu. A. Simonov, Phys. Lett. B 323, 41 (1994); A. Yu. Dubin, A. B. Kaidalov and Yu.A. Simonov, Phys. Atom. Nucl. 56, 1745 (1993).

[21] Yu. S. Kalashnikova, A. Nefediev, Phys. Rev. D 77, 054025 (2008).

[22] L. Brink, P. Di Vecchia, P. Howe, Nucl.Phys. B118, 76 (1977).

[23] Yu. A. Simonov, Phys. Atom. Nucl. 66, 338 (2003) [Yad. Fiz. 66, 363 (2003)].

[24] A. M. Badalian, A. Nefediev, Yu. A. Simonov, Phys. Rev. D78 114020 (2008)

[25] Yu. A. Simonov, Phys. Lett. B 515, 137 (2001).

[26] P. Ball, M. Beneke and V. M. Braun, Nucl. Phys. B452, 563 (1995).

[27] Yu. L. Dokshitzer, G. Marchesini and B. R. Webber, Nucl. Phys. B469, 93 (1996).

[28] see e.g. S. J. Brodsky, S. Menke, C. Merino, and J. Rathsman, Phys. Rev. D67, 055008 (2003) and references therein.

[29] H. Suganuma et al., e-print ArXiv: hep-lat/0407020

[30] H. Leutwyler, Phys. Lett. B 378, 313 (1996), Nucl. Phys. Proc. Suppl., B94, 108 (2001).

[31] E. Gamiz et al., Phys. Rev. Lett. 94, 011803 (2005); S. Narison, Phys. Rev. D 74, 034013 (2006); K.G. Chetyrkin, A. Khodjamiran, Eur. Phys. J. C 46, 721 (2006).

[32] A. M. Badalian, Phys. At. Nuclei 63, 2173 (2001), A. M. Badalian and D. S. Kuzmenko, Phys. Rev. D 65, 016004 (2002).

[33] K.-T. Chao, N. Isgur, and G. Karl, Phys. Rev. D 23, 155 (1981).

[34] O. N. Driga and I. M. Narodetskii, in preparation.

[35] A. De Rujula et al., Phys. Rev. D 12, 147 (1975). 
[36] L. Ya. Glozman and D. O. Riska, Phys. Rep. 268, 263 (1996); L. Ya. Glozman, W. Plessas, K. Varga, and R. F. Wagenbrunn, Phys. Rev. D 58, 094030 (1998).

[37] A. Valcarce, H.Garcilazo, and J. Vijande, Eur. Phys. J. A 37, 217 (2008)

[38] V. Pervin and W. Roberts, Phys. Rev. C77, 025202 (2008)

[39] S. Capstick and N. Isgur, Phys. Rev. D 34, 2809 (1986)

[40] F. X. Lee and X. Liu, Phys. Rev. D 66, 014014 (2002). 